\title{
Frequent and recent retrotransposition of orthologous genes plays a role in the evolution of sperm glycolytic enzymes
}

\author{
Soumya A Vemuganti1,3, Fernando Pardo-Manuel de Villena*2,3,4 and Deborah A O'Brien*1,3,4
}

\begin{abstract}
Background: The central metabolic pathway of glycolysis converts glucose to pyruvate, with the net production of 2 ATP and 2 NADH per glucose molecule. Each of the ten reactions in this pathway is typically catalyzed by multiple isozymes encoded by a multigene family. Several isozymes in this pathway are expressed only during spermatogenesis, and gene targeting studies indicate that they are essential for sperm function and male fertility in mouse. At least three of the novel glycolytic isozymes are encoded by retrogenes (Pgk2, Aldoart1, and Aldoart2). Their restricted expression profile suggests that retrotransposition may play a significant role in the evolution of sperm glycolytic enzymes.
\end{abstract}

Results: We conducted a comprehensive genomic analysis of glycolytic enzymes in the human and mouse genomes and identified several intronless copies for all enzymes in the pathway, except Pfk. Within each gene family, a single orthologous gene was typically retrotransposed frequently and independently in both species. Several retroposed sequences maintained open reading frames (ORFs) and/or provided evidence of alternatively spliced exons. We analyzed expression of sequences with ORFs and $<99 \%$ sequence identity in the coding region and obtained evidence for the expression of an alternative Gpi1 transcript in mouse spermatogenic cells.

Conclusions: Our analysis detected frequent, recent, and lineage-specific retrotransposition of orthologous glycolytic enzymes in the human and mouse genomes. Retrotransposition events are associated with LINE/LTR and genomic integration is random. We found evidence for the alternative splicing of parent genes. Many retroposed sequences have maintained ORFs, suggesting a functional role for these genes.

\section{Background}

Although glycolysis is highly conserved, this central metabolic pathway is modified extensively during spermatogenesis. There are several glycolytic isozymes with restricted expression in the male germline including spermatogenic glyceraldehyde-3-phosphate dehydrogenase (GAPDHS) [1,2], phosphoglycerate kinase 2 (PGK2) [3], and two aldolase A(ALDOA)-related isozymes (ALDOART1 and ALDOART2) in mouse [4]. Other unique sperm isozymes in this pathway are generated by alternative splicing, including hexokinase 1 variants

\footnotetext{
*Correspondence: dao@med.unc.edu, fernando@med.unc.edu

1 Department of Cell and Developmental Biology, University of North Carolina School of Medicine, Chapel Hill, NC 27599, USA

2 Department of Genetics, Carolina Center for Genome Sciences, University of North Carolina School of Medicine, Chapel Hill, NC 27599, USA

Full list of author information is available at the end of the article
}

(HK1_V1 and HK1_V2) [5-7], ALDOA_V2 [4], and a pyruvate kinase muscle form isozyme (PK-S) [8]. There is also evidence that other glycolytic enzymes have unique functional or structural properties in mammalian sperm, including glucose phosphate isomerase (GPI1) $[9,10]$, triose phosphate isomerase (TPI) [11], enolase (ENO) [1214], and phosphofructokinase (PFK) [15].

Sperm motility is dependent upon the production of high levels of ATP in the flagellum [16-18]. Targeted disruption of genes encoding two spermatogenic cell-specific glycolytic enzymes (Gapdhs and $P g k 2$ ) demonstrates an essential role of these enzymes in sperm motility and male fertility in mice $[19,20] . L d h c$, which encodes a germ cell-specific LDH isozyme for the conversion of pyruvate to lactate, is also required [21]. A recent study of 1085 patients with male factor infertility found that approximately $81 \%$ exhibit defects in sperm motility, with $19 \%$ 
having no other defects in sperm count or morphology [22]. The expression of genes that promote high sperm motility can increase reproductive fitness, while disruptive mutations in genes essential for sperm motility can hinder proper fertilization, leading to infertility. In humans, genes involved in spermatogenesis and sperm motility demonstrate the strongest evidence for positive selection, and proteins involved in reproduction are among the most rapidly evolving genes across multiple species $[23,24]$.

The glycolytic pathway is comprised of ten enzymes, each encoded by a multigene family [25]. Seven of these gene families have two to five intron-containing genes, while the Gpi1, Tpi1, and Pgk families each have only one. Within a family, each gene encodes a different isoform with a unique expression pattern [25]. Many of these gene families arose by multiple rounds of segmental gene duplication in the last 150 million years [25]. Genes encoding spermatogenic cell-specific glycolytic isozymes were generated by either segmental gene duplication (Gapdhs) or retrotransposition (Pgk2, Aldoart1, Aldoart2) [3,4,26,27]. Pgk2 represent an ancient retrotransposition event shared by all eutherian mammals, while Aldoart 1 and Aldoart2 are only found in the rodent lineage and are much more recent $[4,28]$. In addition, frequent retrotransposition of the Gapdh and Aldoa genes has been reported in both human and mouse, based on an abundance of pseudogenes [29-32].

Theoretically, retrotransposition can occur in any cell type, but the retrotransposition event is only transmitted to future generations when it takes place in the germline [33-36]. Retrotransposition is facilitated by repetitive elements (including LINE and LTR elements), resulting in the creation of pseudogenes or retrogenes [37]. In the human lineage most LTR elements have been extinct for over 40 million years. However, LINE elements are still active and are, therefore, thought to be responsible for most retroposed mRNA sequences [37]. The proteins encoded by LINE elements provide both endonuclease and reverse transcriptase activities required for retrotransposition. These proteins, ORF1 and ORF2, are expressed in testicular germ cells undergoing meiosis, a period when retrotransposition is thought to occur $[34,36,38]$. In fact, retrotransposition is responsible for the creation of many retrogenes expressed only during the meiotic and/or haploid phases of spermatogenesis, including but not limited to $P g k 2$, G6pd2, and Pabp2 $[3,26,39,40]$. At least $10 \%$ of retroposed sequences with open reading frames (ORF) are transcribed during spermatogenesis [38,41-43]. Positive selection of sperm proteins, combined with frequent retrotransposition to create genes encoding sperm-specific proteins, results in the faster evolution of genes involved in sperm function [44].
Based on the existence of $P g k 2$ and Aldoa-related retrogenes and their restricted expression during spermatogenesis, we hypothesized that there may be additional retrogenes that encode novel sperm glycolytic enzymes. Therefore, we conducted a comprehensive genomic analysis to identify all human and mouse retroposed sequences that are derived from genes encoding glycolytic enzymes. We analyzed the gene structure of these sequences and determined which copies maintain ORFs, are transcribed, and may encode sperm-specific isoforms of glycolytic enzymes. Unique features of sperm glycolytic isozymes may be important for localization of this pathway in the principal piece of the sperm flagellum or for altered regulation or kinetic properties that may be required to sustain sperm metabolism and motility in this highly polarized cell. Taken together, identification of all sperm-specific glycolytic enzymes will improve our understanding of sperm metabolism at a molecular level and may provide insights regarding the rapid evolution of genes required for reproduction.

\section{Results}

Frequent retrotransposition of orthologous genes encoding glycolytic enzymes occurred independently in the mouse and human genomes

There are 25 intron-containing genes in the ten gene families that compose the glycolytic pathway (Table 1). We used BLAST to identify sequences with significant sequence similarity to each parent gene (see Methods for details). This analysis identified retroposed sequences in the human and mouse genomes in every family of glycolytic enzymes, except phosphofructokinase (Pfk). Major conclusions from this analysis are:

- Retrotransposition of genes encoding glycolytic enzymes is frequent. We identified 94 matching retroposed sequences in the human genome and 291 in the mouse genome. Our analysis confirms that the mouse genome contains significantly more retroposed sequences than the human genome [45].

- As a rule, only one gene within each family is retroposed (bolded font in Table 1).

- The same orthologous gene is retroposed in the human and mouse genomes. This is always true in cases where there is more than one retroposed sequence. The two exceptions to this rule, hexokinase $(H k)$ and phosphoglycerate mutase (Pgam), have a single retroposed sequence in one or both species. In the human genome $H K 2$ is retroposed, while $H k 1$ is retroposed in the mouse genome. There is also a single Pgam5 retroposed sequence in mouse in addition to multiple retroposed sequences for Pgam1 in both species.

- The location of retroposed sequences in the human (Additional file 1) and mouse (Additional file 2) 
Table 1: Gene families encoding glycolytic enzymes and the parent genes that are retroposed.

\begin{tabular}{|c|c|c|c|c|c|c|}
\hline \multirow[b]{2}{*}{ Gene Family } & \multicolumn{3}{|c|}{ Human } & \multicolumn{3}{|c|}{ Mouse } \\
\hline & Gene Name & Position & Ret.seq. & Gene Name & Position & Ret.seq. \\
\hline \multirow[t]{5}{*}{ Hexokinase } & HK1 & $10: 71$ & No & $H k 1$ & $10: 62$ & Yes (1) \\
\hline & $H K 2$ & 02:75 & Yes (1) & $H k 2$ & $6: 83$ & No \\
\hline & HK3 & $05: 176$ & No & $H k 3$ & 13:55 & No \\
\hline & GCK & $07: 44$ & No & Gck & $11: 6$ & No \\
\hline & $H K D C 1$ & $10: 71$ & No & $H k d c 1$ & $10: 62$ & No \\
\hline Glucose Phosphate Isomerase & GPI1 & $19: 40$ & Yes (1) & Gpi1 & $7: 34$ & Yes (1) \\
\hline \multirow[t]{3}{*}{ Phosphofructokinase } & PFKL & $21: 45$ & No & $P f k l$ & $10: 77$ & No \\
\hline & PFKM & $12: 47$ & No & $P f k m$ & 15:98 & No \\
\hline & PFKP & $10: 3$ & No & Pfkp & $13: 7$ & No \\
\hline \multirow[t]{3}{*}{ Aldolase } & ALDOA & $16: 30$ & Yes (2) & Aldoa & $7: 127$ & Yes (18) \\
\hline & $A L D O B$ & 09:103 & No & Aldob & $4: 50$ & No \\
\hline & $A L D O C$ & $17: 24$ & No & Aldoc & $11: 78$ & No \\
\hline Triosephosphate isomerase & TPI1 & $12: 7$ & Yes (4) & Tpi1 & $6: 125$ & Yes (15) \\
\hline \multirow[t]{2}{*}{ Glyceraldehyde 3-phosphate dehydrogenase } & GAPDH & $12: 7$ & Yes (52) & Gapdh & $6: 125$ & Yes (188) \\
\hline & GAPDHS & 19:41 & No & Gapdhs & $7: 30$ & No \\
\hline Phosphoglycerate kinase & PGK1 & $\mathrm{X}: 77$ & Yes (3) & Pgk1 & $X: 102$ & Yes (11) \\
\hline \multirow[t]{3}{*}{ Phosphoglycerate mutase } & PGAM1 & 10:99 & Yes (21) & Pgam 1 & $19: 42$ & Yes (12) \\
\hline & PGAM2 & 07:44 & No & Pgam2 & $11: 6$ & No \\
\hline & PGAM5 & $12: 132$ & No & Pgam5 & $5: 111$ & Yes (1) \\
\hline \multirow[t]{4}{*}{ Enolase } & ENO1 & $01: 9$ & Yes (4) & Eno1 & $4: 149$ & Yes (27) \\
\hline & ENO2 & $12: 7$ & No & Eno2 & $6: 125$ & No \\
\hline & ENO3 & $17: 5$ & No & Eno3 & $11: 70$ & No \\
\hline & ENO4 & 10:119 & No & Eno4 & 19:59 & No \\
\hline \multirow[t]{2}{*}{ Pyruvate kinase } & $P K L R$ & $01: 154$ & No & Pklr & $3: 89$ & No \\
\hline & PKM2 & $15: 70$ & Yes (6) & Pkm2 & 9:59 & Yes (17) \\
\hline \multicolumn{7}{|l|}{ Ensembl release 48 Dec 2007} \\
\hline
\end{tabular}


genomes appears to be random. There is no region or chromosome with an overrepresentation of retroposed sequences. There is also evidence for segmental gene duplication of retrotransposed sequences.

- Retrotransposition events occurred independently in each lineage following the divergence of primates and rodents. Phylogenetic analysis was inconclusive in determining the strict order of retrotransposition events due to the high levels of sequence identity between retroposed sequences and parent genes. Analysis of genes flanking retroposed sequences confirmed that these events occurred independently in each species (Additional file 3).

- Human retroposed sequences derived from genes encoding glycolytic enzymes are more divergent from their parent genes than mouse retroposed sequences (Figure 1). Figure 1 groups retroposed sequences matching glycolytic enzymes by the percent nucleotide substitution in the entire sequence (ORFs and UTRs) compared to the parent gene. Human retroposed sequences are $82-100 \%$ identical to their parent genes, with a mean nucleotide identity of $89.2 \%$. Mouse retroposed sequences have the same range of nucleotide identity, although the mean identity is 93.4\%.

\section{3-6\% of retroposed sequences have ORFs}

$6.3 \%$ of human and $3.4 \%$ of mouse retroposed sequences derived from genes encoding glycolytic enzymes contain ORFs equivalent to the full-length ORF present in the parent gene (Figure 2). This value includes the previously identified $P g k 2$, Aldoart1, and Aldoart 2 retrogenes. In this study we identified five new retroposed sequences with ORFs in the human genome and seven new retroposed sequences with ORFs in the mouse genome (Figure 2 , related sequences $[\mathrm{rs}]$ denoted with red and yellow bars representing segments that match exons in the parent gene). Numbers next to each gene structure indicate the percent identity of the coding region in each retroposed sequence compared to the parent gene.

Three of the five human retroposed sequences with ORFs (TPI1-rs1, PGAM1-rs7, ENO1-rs1) showed substantial divergence from their parent genes at both the nucleotide (Figure 2) and amino acid level (Additional file 4). For example, the ORF of PGAM1-rs7 is only $98.3 \%$ identical at the nucleotide level and encodes 11 unique amino acid residues. The mouse sequences we identified are more similar to their parent genes (99.6\% identity) that humans sequences (97.4\%). Six of seven mouse retroposed sequences had ORFs with $>99 \%$ nucleotide and amino acid identity to their parent genes (Figure 2). Two of these sequences, Pgk1-rs1 and Pgk1-rs2, had less than 99\% nucleotide identity in the 3'UTR due to a 5-base-pair insertion that we did not detect in RT-PCR analyses of testis transcripts (data not shown). Gpi-rs1 was the only mouse retroposed sequence that showed less than $99 \%$ sequence identity at the nucleotide (Figure 2) and amino acid level (Additional file 5). These results indicate that several retroposed sequences matching glycolytic enzymes in both the human and mouse genomes have ORFs, supporting possible expression of these sequences.

\section{Detection of splice variants in the glycolytic enzyme parent genes}

Analysis of retroposed sequences derived from glycolytic enzyme genes support the expression of alternative transcripts from the parent genes in both the human and mouse. Two retroposed sequences in humans (ALDOArs 1 and TPI1-rs3) suggest alternative splicing of internal exons (represented as boxes with diagonal lines in Figure 2). For example, TPI1-rs3 is missing 2 full consecutive exons and part of a third, but still contains sequence that
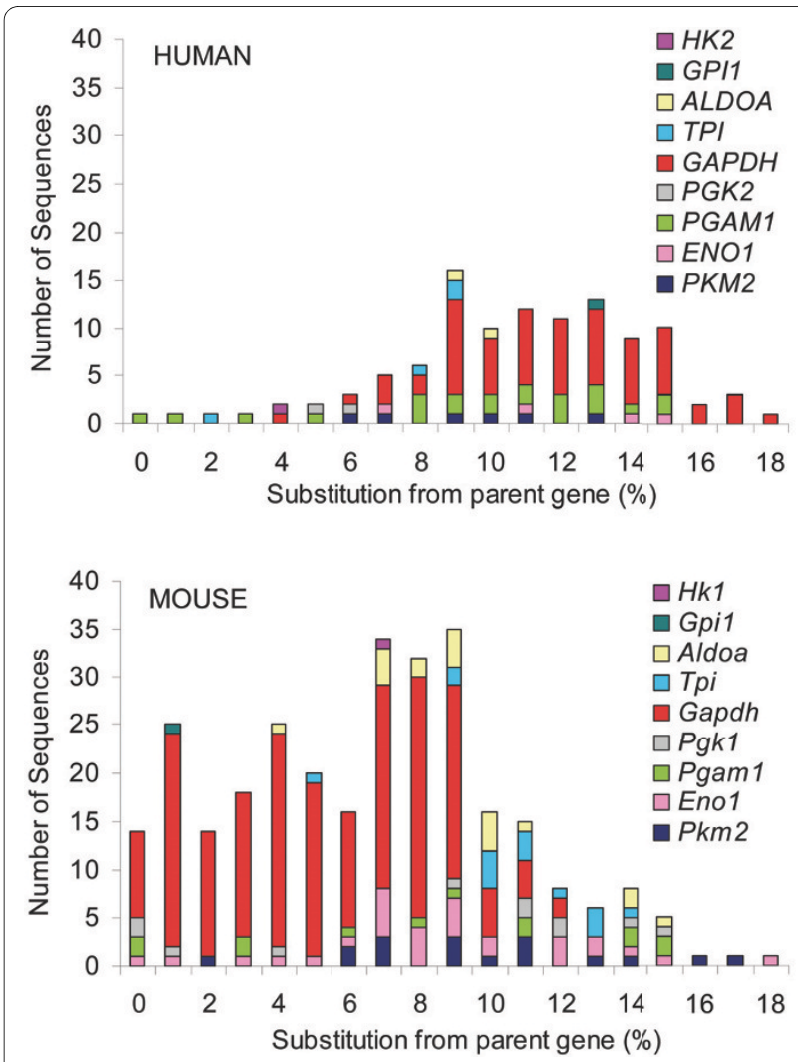

Figure 1 Gene and species-specific divergence of human and mouse retroposed sequences. Percent substitution at the nucleotide level in the entire retroposed sequences (ORFs and UTRs) compared to each parent gene. Retroposed sequences matching each enzyme are represented by a different color, as shown in the inset. Retroposed sequences for each parent gene are shown in the same order as the inset. Each retroposed sequence in represented once at the appropriate substitution level and each bar represents the number of sequences (y-axis) with the percent substitution compared to the parent gene (x-axis). Human sequences are shown on the top and mouse sequences are shown on the bottom. 


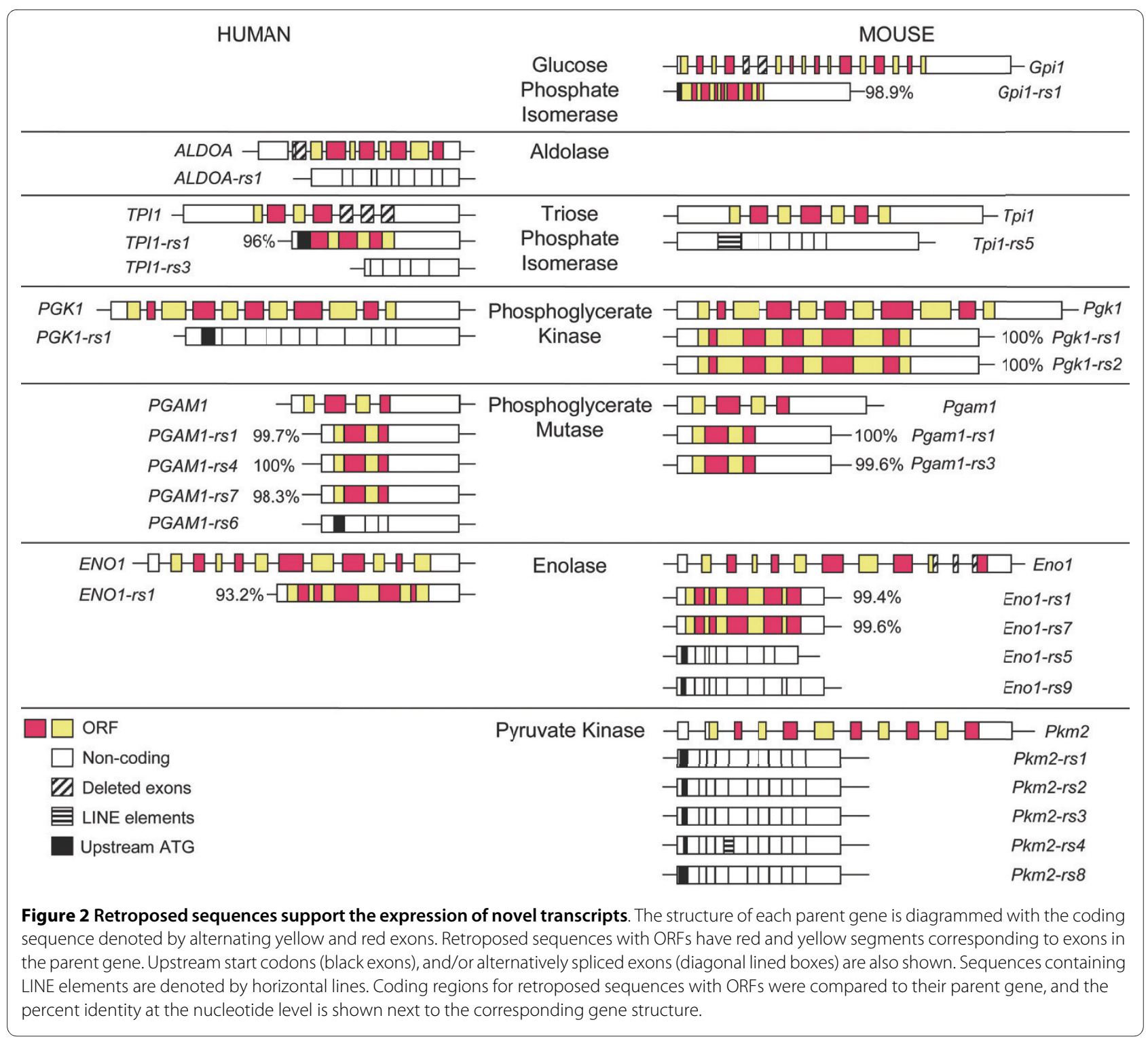

matches part of the last TPI1 exon. Alternative splicing is also supported by two mouse retroposed sequences (Gpi1-rs1 and Eno1-rs5). For example, Eno1-rs5 matches full-length Eno1, except for a deletion of half of exon 10, exon 11 and half of exon 12 (boxes with lines, Figure 2). The remaining 3'UTR is maintained, without the coding sequence of the last exon. However, we did not detect expression of these splice variants in published EST libraries.

\section{Detection of $\mathrm{N}$-terminal extensions in the glycolytic enzyme parent genes}

Some spermatogenic-cell specific glycolytic enzymes are modified through the addition of amino acid residues at the N-terminus, including GAPDHS, ALDOA_V2 and ALDOART1 [2,4]. Our previous analysis supported the retrotransposition of an alternative splice variant
(Aldoa_v2) to produce a novel gene encoding an N-terminal extension (Aldoart1) [4]. In this study, we found that multiple mouse and human retroposed sequences have upstream start codons, supporting the expression of transcripts that encode additional glycolytic enzymes with $\mathrm{N}$ terminal extensions. Three human sequences (TPI1-rs1, PGK1-rs1 and PGAM1-rs6) and nine mouse sequences (Gpi1-rs1, Tpi1-rs5, Eno1-rs5, 9, and Pkm2-rs1, 2, 3, 4, 8) contain upstream start codons (black exons, Figure 2). In most cases, comparison of the amino acid sequence in these $\mathrm{N}$-terminal extensions reveal a unique origin for these extensions that is independent from the parent genes (Additional file 6), since the alignment does not show a high level of identity. Five retroposed sequences matching $P k m 2$ in mice contain N-terminal extensions. Previous studies detected a larger $P k m 2$ protein in boar 
and mouse sperm $[8,46]$. Proteomic evidence from boar sperm suggests extension of the $\mathrm{N}$-terminus by at least five amino acids [8]. Our sequence analysis of $P k m 2$ retropseudogenes with upstream start codons in the mouse genome shows partial agreement with the previously identified five-amino-acid extension, but does not clearly elucidate the start codon responsible for the larger protein product detected in sperm (Additional file 6).

\section{Novel ORFs with divergent sequences are not expressed in human testis}

Our expression analyses in both species focused on retroposed sequences with less than $99 \%$ identity at the nucleotide level and did not include the large number of sequences derived from GAPDH. Due to high sequence similarity at the nucleotide level, we used Single Strand Conformation Polymorphism (SSCP) gel electrophoresis to examine potential expression of three retroposed sequences in human testis (Figure 3A). In this method RT-PCR products are denatured into two strands and separated based upon individual nucleotide differences, allowing us to distinguish between sequences with very high levels of identity. Expression of protamine 1 (PRM1), a spermatid-specific transcript, was detected in human cDNA preparations, confirming complete spermatogenesis in the pooled testes tissues used for RNA isolation. We used genomic DNA to identify the migration pattern of the PCR products amplified from the retroposed sequences (G1 and G2, Figure $3 \mathrm{~B}$ ). With primers specific for TPI-rs1, PGAM1-rs7, and ENO1-rs1, RT-PCR did not amplify products from human testis RNA that match the retroposed sequence fragments amplified from genomic DNA (Figure 3B). Therefore, we did not detect testis expression of the human retroposed sequences with ORFs that were analyzed in this study.

\section{Expression of an alternative Gpi 1 transcript in mouse spermatogenic cells}

Gpi1-rs1 maintains an ORF, despite missing two internal exons (Figure 4A). The open reading frame of Gpi1-rs1 suggests the expression of this retroposed sequence and/ or a Gpi1 splice variant missing exons 5 and 6 (Gpi1_v2). RT-PCR expression analysis in mouse tissues revealed a testis-specific transcript of glucose phosphate isomerase, representative of Gpi1_v2 and/or Gpi1-rs1 (Figure 4B). This transcript was also detected in both pachytene spermatocytes and round spermatids isolated from mouse testis, but not in later germ cells (condensing spermatids) or Sertoli cells. The same band was detected in human testis, but due to the absence of Gpi1-rs1 in the human genome, must represent the expression of GPI1_V2 (data not shown).

To distinguish between Gpi1_v2 and Gpi1-rs1 expression, we designed PCR primers to specifically detect

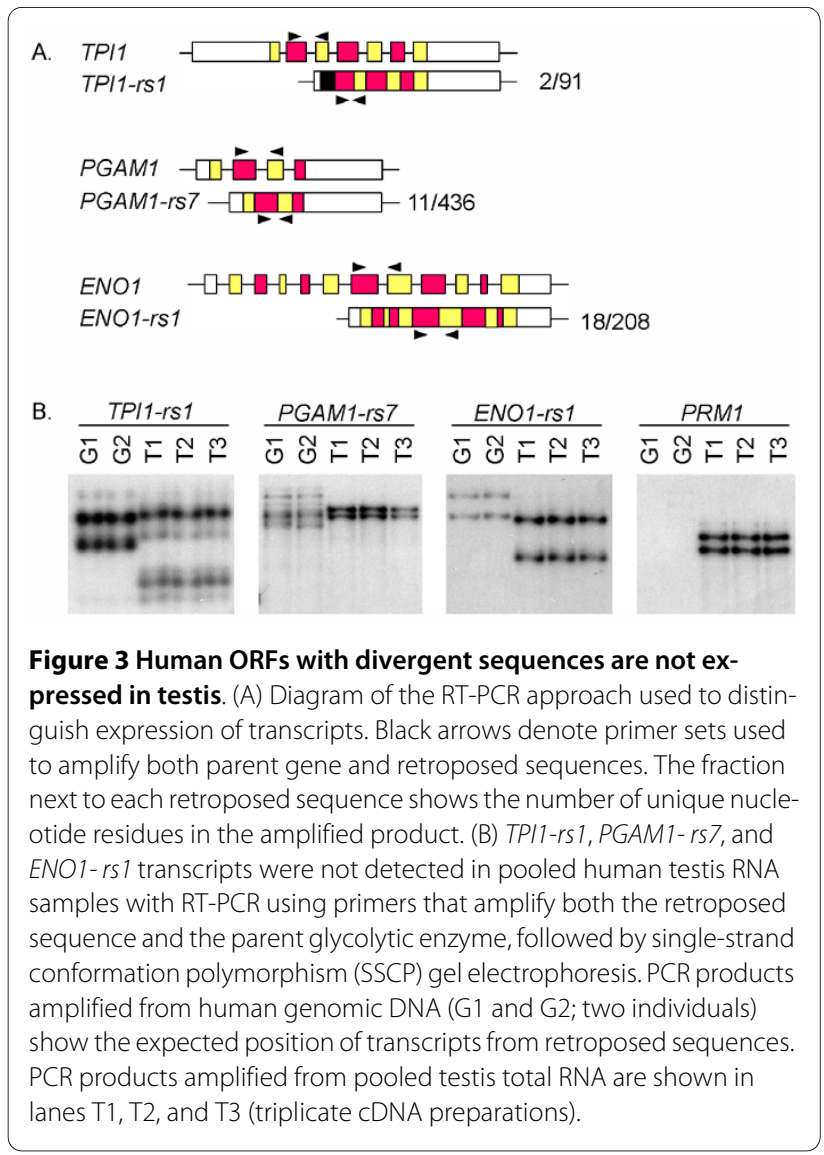

expression of Gpi1-rs1 (Figure 4A). Using this approach, we did not detect a Gpi1-rs1-specific product (Figure 4B, bottom panel), indicating that PCR products initially detected in pachytene spermatocytes and round spermatids (Figure 4B, top panel) are most likely derived from Gpi1_v2 transcripts.

We detected expression of the GPI1 protein in various tissues and germ cells isolated from mouse testis (Figure 4C). GPI1 has 553 amino acids, while GPI1_V2 has 476 amino acids since it is missing sequence encoded by exons 5 and 6 (Additional file 5). GPI1-rs1 is also missing sequence encoding exons 5 and 6 but contains an $\mathrm{N}$-terminal extension and is, therefore, 485 amino acids (Additional file 5). The predicted molecular weights of GPI1, GPI1_V2, and GPI1-rs1 are 62,800, 54,500 and 55,100, respectively. We detected a protein band that migrates with an apparent molecular weight of $\sim 55,000$ in all tissues analyzed. This band is assumed to be GPI1 due to its ubiquitous expression pattern. We also identified a larger immunoreactive band that was seen only in isolated spermatogenic cells (Figure 4C). This protein is not present in human or mouse sperm and is larger than the predicted molecular weights of GPI1_V2 and GPI1-rs1. We also found that glucose phosphate isomerase is soluble in the supernatant fraction following sonication of mouse 


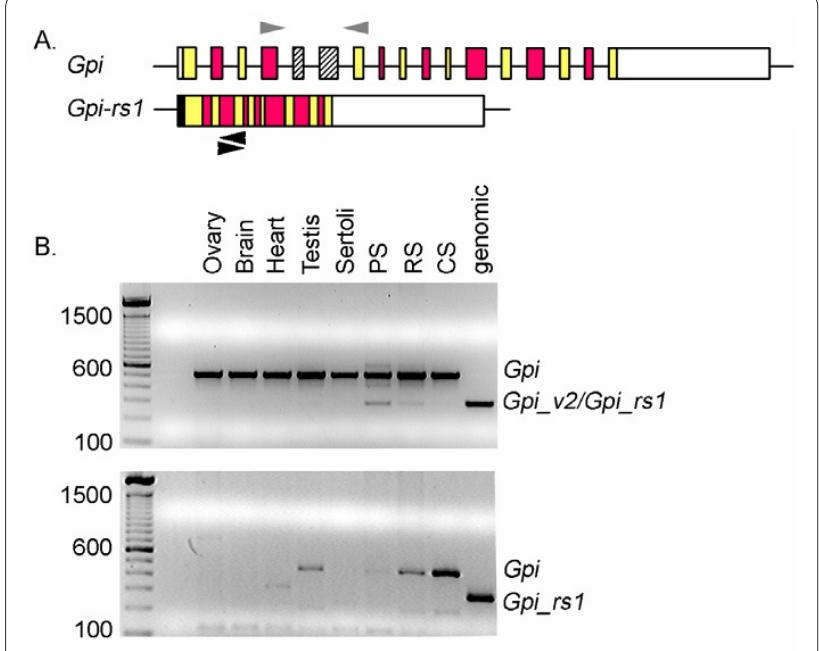

C.

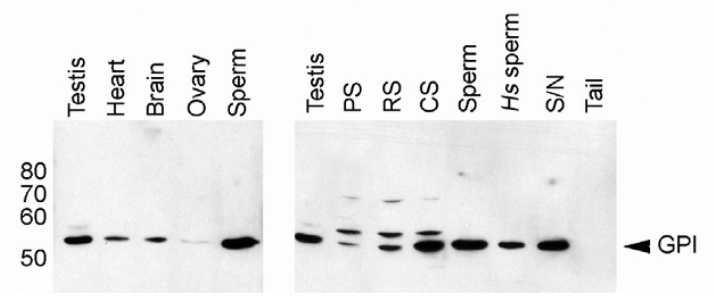

Figure 4 Expression of an alternative Gpi1 transcript in mouse spermatogenic cells. (A) Diagram of RT-PCR approach used to distinguish expression of Gpil-related transcripts. Gray arrows denote the primer set used to differentiate transcripts containing alternatively spliced exons 5 and 6 (boxes with diagonal lines). Black arrows denote the Gpi1-rs1-specific primer set. (B) Transcripts from Gpi1 were detected in all mouse tissues and isolated testicular cells. Gpi1-rs 1 was amplified from genomic DNA to identify the expected size of PCR products from Gpil transcripts not containing exons 5 and 6 . A product of the same size was detected in isolated pachytene spermatocytes (PS) and round spermatids (RS), but not condensing spermatids (CS). This PCR fragment appears to be derived from Gpi1_v2, since Gpi1_rs1-specific primers did not amplify a product. (C) A smaller GPI1_V2 protein was not detected by western analysis using a polyclonal antibody raised against human GPI1. A larger protein product was seen in isolated testicular cell, but not in mouse or human $(\mathrm{Hs})$ sperm. S/N fraction contains proteins solubilized from sperm tail following brief sonication and centrifugation. Tail fraction contains proteins left insoluble following sonication and centrifugation.

sperm (S/N, Figure 4C). Since GPI1 is not found in insoluble fractions of mouse sperm, it is not tightly bound to the fibrous sheath, the cytoskeletal structure in the sperm flagellum that binds multiple glycolytic enzymes with unique $\mathrm{N}$-terminal extensions [4]. Although we were unable to distinguish GPI1_V2 in our Western analysis, we identified an alternative splice variant of Gpil that is transcribed in spermatogenic cells of the mouse testis.

\section{Repetitive elements are overrepresented in sequence flanking retroposed sequences}

The Aldoart1 sequence provided evidence for an alternative splice variant (Aldoa_v2) of aldolase A that is also expressed during spermatogenesis [4]. In our analyses of other retroposed sequences, we examined flanking sequences for evidence of alternative splicing or additional coding sequence, particularly at the $\mathrm{N}$-terminus. Analysis of $1 \mathrm{~kb}$ sequence both upstream and downstream of all human and mouse retroposed sequences did not identify additional coding sequences. Instead, we found a significant $(p<0.01)$ increase in the number of repetitive elements, particularly LINE and LTR elements, in regions that flank retroposed sequences compared to the regions that flank the parent genes (intron-containing genes encoding the glycolytic enzymes) (Additional file 7).

We calculated the percent frequency of both LINE and LTR elements at each base pair within $1 \mathrm{~kb}$ upstream and downstream of each retroposed sequences, as compared to intron-containing parent genes encoding glycolytic enzymes. We observed an increase in LINE and LTR elements along the entire $1 \mathrm{~kb}$ immediately upstream or downstream of retroposed sequences (Figure 5A). Because LINE elements are found preferentially in $(\mathrm{A}+$ $\mathrm{T})$-rich regions of the genome [45], we expected a low ( $\mathrm{G}$ $+C)$ content in the flanking regions $(10 \mathrm{~kb})$ of retroposed sequences. Surprisingly, we found that the $(G+C)$ content matched the $(G+C)$ content of the entire genome for both species (Figure 5B). Therefore, these retroposed sequences and flanking repetitive elements are not preferentially located in $(A+T)$ rich regions. We dated the repeated elements by comparing their nucleotide divergence from their respective consensus sequences, and then compared these values to the nucleotide divergence of the corresponding retroposed glycolytic sequence from the corresponding parent gene (data not shown). We find that there is no correlation.

\section{Discussion}

We found frequent retrotransposition of one member in each gene family encoding the glycolytic enzymes. Remarkably, the orthologous gene is retroposed independently in the human and mouse genomes. Phylogenetic analyses indicate that the retroposed parent gene is not the most slowly-evolving gene in each gene family $[25,31]$. In support of this conclusion, phylogenetic trees for five representative glycolytic enzymes are shown in Additional file 8. Although at least one retrogene, $P g k 2$, is present in all eutherian mammals [47], most of the retroposed sequences identified in this analysis arose following the primate-rodent split. Many factors may contribute to preferential retrotransposition of a single orthologous gene in each gene family in both species.

Germline expression of the parent gene is required for retrotransposition events that can be transmitted to future generations. Retroposed parent genes in the glycolytic pathway are expressed in testicular germ cells, 


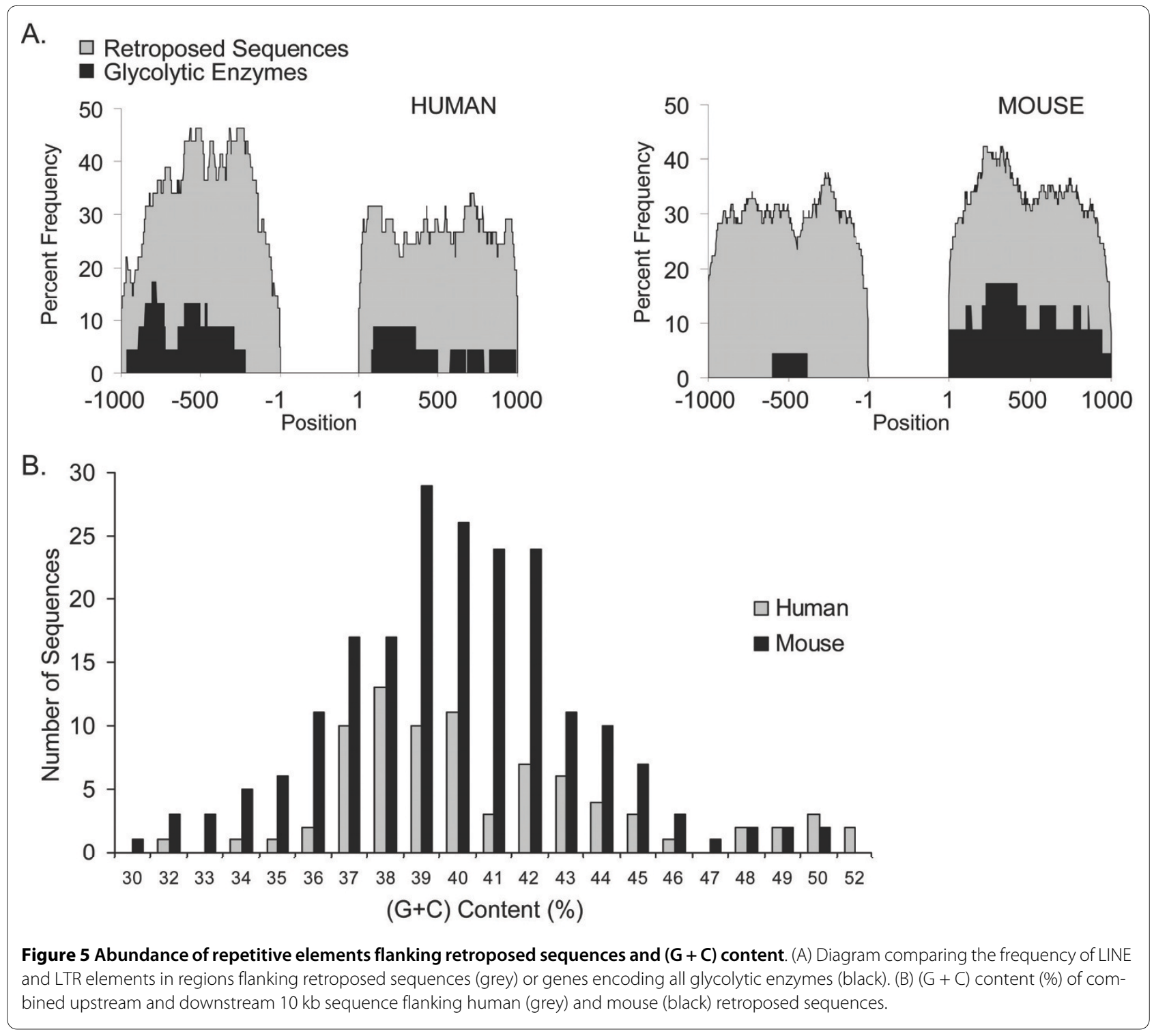

including Hk1, Gpi1, Aldoa, Tpi1, Gapdh, Pgk1, Pgam1, Eno1 and Pkm2 [2,4,7,9,11,48-51]. These studies and microarray analyses of isolated spermatogenic cells http:/ /mrg.genetics.washington.edu/[52] indicate that glycolytic enzyme genes that are retroposed (Table 1) are expressed during early mitotic (spermatogonia) and/or meiotic (spermatocytes) stages of spermatogenesis in the mouse. The same microarray studies indicate that several glycolytic enzyme genes that are not retroposed are not expressed during spermatogenesis (Hk3, Aldob, Aldoc, Eno2 and $P k l r)$ or are expressed only during the haploid period (Gapdhs). mRNA abundance and stability, as well as short length and nucleotide sequence, may contribute to preferential retrotransposition [53-55],

Along with expression of the parent gene, the machinery that facilitates retrotransposition of mRNA transcripts must also be expressed in the same developmental stage/cell type of the germline. ORF1 and ORF2, the LINE element-encoded proteins that provide RNA-binding and enzymatic activities required for retrotransposition, are expressed in testicular germ cells, including preleptotene and zygotene spermatocytes [34,36]. We found an overrepresentation of LINE and LTR repetitive elements flanking retroposed sequences derived from genes encoding glycolytic enzymes, but no evidence that both events occurred simultaneously. These retroposed sequences and flanking repetitive elements are not found in $(\mathrm{A}+\mathrm{T})$ rich regions, where LINE and LTR elements are normally found [45], suggesting a distinct mechanism for the genomic integration of these sequences compared to repetitive elements alone.

Our genomic analyses of retroposed sequences identified Aldoart1 and Aldoart2, two newly identified retrogenes in mouse [4], and may provide additional insights 
regarding the process of retrotransposition and the evolution of expressed retrogenes. We determined that the gene families encoding glycolytic enzymes have single members that are frequently retrotransposed, resulting in the creation of multiple gene copies in the genome. This high frequency suggests that most mammalian species, including those that are phylogenetically close, are likely to differ in the number and function of glycolytic enzymes. Some of the retroposed sequences have ORFs, due either to very recent retrotransposition events providing very little time for the accumulation of sequence divergence, or to selective pressure exerted on sequences that are expressed. Sequences with ORFs that are expressed in testicular germ cells may be acted upon by selective pressure in the context of reproductive fitness, and it is known that there is rapid evolution of genes involved in reproduction [24].

It is well known that gene duplication, including retrotransposition, provides the opportunity for the duplicated genes to diverge by mutation and eventually change or acquire new functions. In contrast with other tissues, the evolution of the glycolytic pathway in spermatogenic cells is focused on insuring the production of high levels of ATP in the sperm flagellum. There are a surprising number of glycolytic variants in mammalian sperm, and recent studies continue to uncover new enzymes and regulatory features of both glycolytic and other metabolic enzymes. For example, it is now clear that glycolysis and respiration occur in two distinct compartments of the sperm flagellum, adding complexity to the typical regulatory interactions of these metabolic pathways [56]. Furthermore, at least five glycolytic enzymes are tightly bound to the fibrous sheath, a cytoskeletal structure in the principal piece of the flagellum $[4,46]$. These include multiple germ cell-specific isozymes with novel N-terminal extensions that are hypothesized to play a role in localizing glycolysis in the principal piece, thereby insuring an adequate supply of ATP along the full length of the flagellum. Adaptations during the ongoing evolution of glycolysis have involved the rapid emergence of new genes by duplication and retrotransposition, the acquisition of distinct expression patterns in male germ cells, changes in the enzymatic properties, and novel structural features that facilitate compartmentalization of the glycolytic pathway in the principal piece of the sperm flagellum.

\section{Conclusions}

We identified frequent retrotransposition in both the human and mouse genomes of a single orthologous gene in each gene family encoding glycolytic enzymes. Integration of retrotransposition events is random. Many of these retroposed sequences have ORFs and some support the expression of alternative splice variants and $\mathrm{N}$-termi- nal extensions. These sequences are lineage-specific and many are recent, indicating that similar genomic analyses in other organisms may identify additional genes encoding glycolytic enzymes. Several of the retroposed sequences identified in this study have retained ORFs despite substantial sequence divergence, suggesting functional importance. Glycolysis is essential for sperm motility and fertilization. It is likely that selective pressure in the context of reproductive fitness contributes to the evolution of novel isozymes in this pathway.

\section{Methods}

\section{Identification of gene families}

The Ensembl http://www.ensembl.org Interpro Domain was used to identify the intron-containing genes for each glycolytic enzyme [57]. We used Ensembl release 48 (Dec 2007) to identify all genes, their mRNA sequences and chromosome locations. Accession numbers used for BLAST searches are:

Human: HK1 [ENSG00000156515] HK2 [ENSG000001 59399] HK3 [ENSG00000160883], GCK [ENSG000001 06633], HKDC1 [ENSG00000156510], GPI1 [ENSG00000 105220], PFKL [ENSG00000141959], PFKM [ENSG000 00152556], PFKP [ENSG00000067057], ALDOA [ENSG0 0000149925], $A L D O B$ [ENSG00000136872], ALDOC [EN SG00000109107], TPI1 [ENSG00000111669], GAPDH [E NSG00000111640], GAPDHS [ENSG00000105679], PG K1 [ENSG00000102144], PGAM1 [ENSG0000017 1314], PGAM2 [ENSG00000164708], PGAM5 [ENSG00000 176894], ENO1 [ENSG00000074800], ENO2 [ENSG000 00111674], ENO3 [ENSG00000108515], DKFZp781N 1041 [ENSG00000188316], PKLR [ENSG00000143627], PKM2 [ENSG00000067225].

Mouse: Hk1 [ENSMUSG00000037012], Hk2 [ENSMUSG00000000628], Hk3 [ENSMUSG000000258 77], Gck [ENSMUSG00000041798], $H k d c 1$ [ENSMUSG0 0000020080], Gpi1 [ENSMUSG00000036427], Pfkl [EN SMUSG00000020277], Pfkm [ENSMUSG00000033065], Pfkp [ENSMUSG00000021196], Aldoa [ENSMUSG00000 030695], Aldob [ENSMUSG00000028307], Aldoc [ENSMUSG 00000017390], Tpi1 [ENSMUSG00000023456], Gapdh [ENSMUSG00000057666], Gapdhs [ENSMUSG 00000061099], Pgam1 [ENSMUSG00000011752], Pgam2 [ENSMUSG00000020475], Pgam5 [ENSMUSG0000002 9500], Eno1 [ENSMUSG00000063524], Eno2 [ENSM USG00000004267], Eno3 [ENSMUSG00000060600], 64 30537HO7Rik [ENSMUSG00000048029], Pklr [ENSMUS G00000041237], Pkm2 [ENSMUSG00000032294].

\section{BLAST search for retroposed sequences}

We blasted the mRNA sequence for each gene encoding a glycolytic enzyme using Ensembl BlastView in order to identify retroposed sequences in both the human and mouse genomes. We grouped BLAST hits based upon 
chromosome location and orientation. Multiple hits in close vicinity and with the same orientation were grouped together in a single hit that span the entire genomic sequences between and upstream/downstream of hits. Hits with less 50 base pairs were excluded. By comparing BLAST results between gene family members, we identified the parent gene for each retroposed sequence. For each retroposed sequence, we identified the parent gene by choosing matches with the longest hit and the highest percentage match. Using the BLAST results, we calculated the weighted average of the nucleotide identity of all retroposed sequences matching glycolytic enzymes. Ensembl was used to retrieve the FASTA sequence for each retroposed sequence on the appropriate strand.

\section{Sequence alignment}

All retroposed sequences were aligned with their parent gene with Sequencher 4.8 (Gene Codes Corporation, Ann Arbor, MI). We used large gap parameters and a $60 \%$ identity threshold to align all sequences to the reference sequence (the parent gene). We examined the nucleotide sequence corresponding to the exons of the parent gene and identified insertions, deletions, and base pair substitutions. We then calculated the percent identity of the coding sequence and looked for an ORF. Amino acid sequence alignments were performed using ClustalW http://www.ebi.ac.uk/Tools/clustalw2/index.html[58].

\section{Tissue and cell isolations}

Outbred CD-1 mice were obtained from Charles River (Raleigh, NC). All procedures involving animals were approved by the University of North Carolina at Chapel Hill Animal Care and Use Committee and conducted in accordance with the Guide for the care and Use of Laboratory Animals (Institute for Laboratory Animal Research, National Academy of Sciences).

All tissues were quick frozen in liquid nitrogen and kept at $-80^{\circ} \mathrm{C}$ until use. Testicular germ cells were isolated using an established protocol [59]. Briefly, we purified pachytene spermatocytes, round spermatids, and condensing spermatids by unit gravity sedimentation from adult mixed germ cell suspensions [59]. Pachytene spermatocytes and round spermatids have purities $>90 \%$, while condensing spermatids have $30-40 \%$ nucleated cells and cytoplasts derived from the same cells. Testes from 17-day-old mice were used to isolate Sertoli cells, as previously described [60].

Mouse sperm was collected as previously described [4]. Briefly, each cauda epididymis was clipped and incubated for 15 minutes at $37^{\circ} \mathrm{C}$ in phosphate-buffered saline with protease inhibitors (PBS + PI) containing $140 \mathrm{mM} \mathrm{NaCl}$, $10 \mathrm{mM}$ phosphate buffer (pH 7.4) and Complete protease inhibitor cocktail (Roche Diagnostics, Mannheim, Ger- many). Cryopreserved human sperm samples from healthy donors were obtained from the Andrology Laboratory, Department of Obstetrics and Gynecology, University of North Carolina School of Medicine. These samples were washed twice with PBS to remove seminal plasma.

\section{RT-PCR expression analysis of newly identified retroposed sequences in mouse and human tissues and cells}

Total RNA was isolated using Trizol (Invitrogen, Carlsbad, CA) from isolated testicular cells or tissues pooled from at least three mice. Adult tissues included brain, heart, ovary and testis. The Qiagen RNeasy Midi Kit (Qiagen Incorporation, Valencia, CA) was used to remove genomic DNA contamination from RNA preparations. RNA was quantified using the NanoDrop spectrophotometer (NanoDrop Technologies, Wilmington, DE). Human RNA prepared from tissues pooled from 39 individuals was purchased from Clontech (Mountainview, CA). Genomic DNA isolated from CD-1 mice and two human subjects were used as positive controls for detected of PCR-amplified retrogenes. Reverse transcription followed by gene-specific polymerase chain reaction (Superscript RT II, Invitrogen, Carlsbad, CA; Taq DNA polymerase, New England BioLabs, Ipswich, MA) was used to amplify transcripts from total RNA samples.

Two primer pairs were designed to detect expression of Gpi1-related transcripts in mouse RNA samples. The first primer pair distinguishes between transcripts that contain alternatively spliced exons 5 and 6 (Figure 4A): Gpi1F in exon 4 (5'GAGGTGAACAGGGTTCTGGA3'), Gpi1R in exon 11 (5'GCTCGAAGTGGTCAAAACC3'). The expected product sizes are as follows: Gpi1, 520 base pairs; Gpi1_v2/Gpi1-rs1, 288 base pairs. The second primer pair is specific for Gpi1-rs1F in exon 4 (5'ATCAAGGTGGTCGGG3'), Gpi1-rs1 $\mathrm{R}$ in exon 10 (5'CAATGGAAGGTCCAG3'). We also included a negative control with no reverse transcriptase as a control for genomic DNA contamination. All PCR products were resolved by $2 \%$ agarose gel electrophoresis and visualized by ethidium bromide staining using UV detection.

To detect expression of human retroposed sequences, primers were designed to amplify and incorporate $\alpha$ [ $\left.{ }^{32} \mathrm{P}\right]-\mathrm{dCTP}$ into the same size product from the parent gene and the retroposed sequences of interest: Tpil/ Tpi1-rs1, Pgam1/Pgam1-rs1, and Eno1/Eno1-rs1. The forward primer sequence for Tpi1/Tpi1-rs1 was 5'CCGACACCGAGGTGGTTT3' and the reverse primer sequence was 5'GTTCTGCGCAGCCACAGCAA3'. The forward primer sequence for Pgam1/Pgam1-rs1 was 5'GCAGACCTCACAGAAGATCAG3' and the reverse primer sequence was 5'ACAGATGTGGTCAGTGTGACA T3'. The forward primer sequence for Eno1/Eno1rs1 was 5'TTGGGAAAGCTGGCTACACT3' and the 
reverse primer sequence was 5' CCAGTCATCCTGGTCAAAGG 3'. Arrows in Figure 3A denote the location of these primers in each gene.

As a positive control to confirm proper spermatogenesis in human testis samples, we detected expression of protamine 1 (Prm1) in RNA samples. We also included a negative control with no reverse transcriptase as a control for genomic DNA contamination. The forward primer sequence for Prm1 was 5'TCACAGGTTGGCTGGC TC3'and the reverse primer sequence was 5'CATTGTTCCTTAGCAGGCTCC3' [61]. Following PCR amplification with both primer sets, the products were resolved by Single Strand Conformation Polymorphism (SSCP) electrophoresis using MDE gel solution (Cambrex, East Rutherford, NJ) at $0.5 \mathrm{~W}$ for 19 hours. Genomic DNA was used as a control template in parallel PCR reactions to confirm the expected electrophoretic pattern of the retroposed sequences. Gels were exposed to Super RX X-ray film (Fujifilm, Tokyo, Japan) using intensifying screens to detect incorporation of $\alpha$-[32P]dCTP into amplified products.

\section{Western analysis of GPI1-related proteins}

Lysis buffer (2\% SDS, 100 mM DTT, 125 mM Tris pH 6.8, $18 \%$ glycerol) was used to extract proteins from tissues or isolated cells. Samples were centrifuged at $16,000 \times g$ for $10 \mathrm{~min}$ at $4^{\circ} \mathrm{C}$ following homogenization. Protein concentrations were determined using the micro-BCA assay (Pierce Biotechnology, Rockford, IL). SDS polyacrylamide gel electrophoresis (SDS-PAGE) on 7.5\% polyacrylamide gels was used to separate samples with equal protein amounts, followed by electrophoretic transfer to Immobilon-P PVDF (polyvinylidene fluoride) membranes (Millipore Corp, Bedford, MA). Equal protein loading was confirmed by Coomassie blue R250 staining ( $0.1 \%$ Coomassie blue R250 in 45\% methanol, $10 \%$ acetic acid). Membranes were destained, rinsed with TBS-T $(140 \mathrm{mM}$ $\mathrm{NaCl}, 3 \mathrm{mM} \mathrm{KCl}, 0.05 \%$ Tween-20, $25 \mathrm{mM}$ Tris-HCl, pH 7.4) and incubated in blocking buffer (5\% nonfat dry milk in TBS-T) overnight at $4^{\circ} \mathrm{C}$. Antibody incubations were performed at room temperature in blocking buffer. Membranes were incubated with a 1:500 dilution of a polyclonal antibody raised against a recombinant human glucose phosphate isomerase protein fragment (Strategic Diagnostic Incorporation, Newark, DE) for 2 hours. Membranes were then incubated for $45 \mathrm{~min}$ at room temperature with secondary antibody (affinity-purified horseradish peroxidase-conjugated rabbit anti-goat IgG, KPL, Gaithersburg, MD) diluted 1:10,000. Following antibody incubations, membranes were rinsed for 5 minutes with TBS-T. Immunoreactive proteins were detected by enhanced chemiluminescence using the SuperSignal West Pico substrate (Pierce Biotechnology, Rockford, IL) and HyBlot $\mathrm{CL}$ autoradiography film (Denville Scientific, Metuchen, NJ).

\section{Repetitive Element Analysis}

Galaxy http://galaxy.psu.edu was used to obtain both $1 \mathrm{~kb}$ and $10 \mathrm{~kb}$ FASTA format sequence flanking retroposed sequences and genes encoding glycolytic enzymes [62]. We analyzed FASTA format sequence for all retroposed sequences, $1 \mathrm{~kb}$ flanking retroposed sequences and $1 \mathrm{~kb}$ flanking genes encoding glycolytic enzymes for the presence of repetitive elements using Repeatmasker http:// www.repeatmasker.org. We calculated the percent frequency of repetitive elements (LINE, LTR, and SINE) in each base pair within $1 \mathrm{~kb}$ upstream or downstream of retroposed sequences or genes encoding glycolytic enzymes. Chi-square values were calculated using a contingency table comparing mouse and human sequences versus glycolytic enzymes and retroposed sequences for each repetitive element. $(G+C)$ content was calculated using the eMBOSS geecee program http://inntemp.weizmann.ac.il/cgi-bin/emboss/geecee[63].

\section{BLAST search for extensions}

Repeatmasker http://www.repeatmasker.org was used to generate sequence with the repetitive elements masked (represented by "n") [64]. We repeatmasked the $1 \mathrm{~kb}$ sequence flanking all retroposed sequences and used Ensembl BLAST to compare this sequence to the mouse or human genome. We looked for matches with genomic locations close to either the parent gene or other retroposed sequences, indicative of a sequence extension at the end of the gene.

\section{Dating retroposed sequences}

We dated the repeated elements by comparing their nucleotide divergence from their respective consensus and then compared these values to the nucleotide divergence of the corresponding retroposed glycolytic sequence to the corresponding parent gene. In addition, we determined whether retroposed sequences are located at homologous position of the human and mouse genome by determining the position of the flanking genes in the appropriate species. We then found the position of the homologous genes in the others species using comparative maps http://www.ncbi.nlm.nih.gov/projects/homology/maps/. Finally, to determine the evolutionary history of genes within each gene family and their rate of divergence we aligned the coding sequence using ClustalW http://www.ebi.ac.uk/Tools/clustalw2/index.html[58] and constructed a distance tree using the Neighbor Joining method from the PHYLIP package http://evolution.genetics.washington.edu/phylip/.

\section{List of Abbreviations}

Hk: Hexokinase; Gpi1: Glucose phosphate isomerise; Pfk: Phosphofructokinase; Aldo: Aldolase; Tpi1: Triose phosphate isomerise; Gapdh: Glyceraldehyde phosphate dehydrogenase; Pgk: Phosphoglycerate kinase; Pgam: 
Phosphoglycerate mutase; Eno: Enolase; Pk: Pyruvate kinase; Ldh: Lactate dehydrogenase; LINE: Long interspersed nucleotide element; LTR: Long terminal repeats; SINE: Short interspersed nucleotide element; SSCP: Single strand conformation polymorphism electrophoresis; ATP: Adenosine triphosphate; RT-PCR: Reverse transcription polymerase chain reaction; ORFs: open reading frames.

\section{Additional material}

\section{Additional file 1 Human retroposed sequences matching genes} encoding glycolytic enzymes. This table indicates the gene name for each retroposed sequence in the human genome, along with the chromosome position and strand. FL CDS refers to those sequences containing fulllength coding sequence $(Y)$ or only untranslated sequence (UTR), regardless of whether the sequences are in frame. In some cases, multiple retroposed sequences from the same parent gene are located at adjacent chromosome positions. For example ENO1-rs2 and ENO1-rs3 are located less than $2 \mathrm{~kb}$ apart on chromosome 15

Additional file $\mathbf{2}$ Mouse retroposed sequences matching genes encoding glycolytic enzymes. This table indicates the gene name for each retroposed sequence in the mouse genome, along with the chromosome position and strand. FL CDS refers to those sequences containing fulllength coding sequence $(Y)$ or only untranslated sequence (UTR), regardless of whether the sequences are in frame. In some cases, multiple retroposed sequences from the same parent gene are located at adjacent chromosome positions. For example: Eno1-rs15 and Eno1-rs23 are located less than $1 \mathrm{~kb}$ apart on chromosome $3 \mathrm{~m}$ and Pkm2-rs2 and Pkm2-rs3 are located $150 \mathrm{~kb}$ apart on chromosome 2 .

\section{Additional file 3 The position of genes flanking retroposed} sequences in the mouse and human genome. The table identifies the genes flanking each of the retroposed sequences derived from genes encoding glycolytic enzymes. For each retroposed sequenced we determined the position of the flanking genes in the appropriate species and determined the position of the homologous genes in the others species using well established comparative maps http://www.ncbi.nlm.nih.gov/ projects/homology/maps/.

Additional file 4 Amino acid alignment of retroposed sequences in the human genome that maintain ORFs. Asterisks $\left(^{*}\right)$ denote identical residues. Methionine residues are highlighted in grey boxes, residues marked as "X" in a black box denote stop codons, and dashes denote deleted codons.

Additional file 5 Amino acid alignment of GPI1-related sequences in the mouse genome that maintain ORFs (GPI1-rs1). Asterisks $\left(^{*}\right)$ denote identical residues. Methionine residues are highlighted in grey boxes, dashes denote deleted codons and the stop codon is marked as "X" in a black box.

Additional file $\mathbf{6}$ Amino acid alignments are shown for retroposed sequences containing upstream start codons. (A) Amino acid sequence alignment comparing upstream extensions of human retroposed sequences to their parent glycolytic enzymes. (B) Amino acid sequence alignment of mouse retroposed sequences with upstream start codons and their parent glycolytic enzymes. Asterisks $\left(^{*}\right)$ denote identical residues. Methionine residues are highlighted in grey boxes, residues marked as "X" in a black box denote stop codons, and dashes indicate deleted codons. Pkm2-Ss sequence is from [8]. Hs (Homo sapiens), Bt (Bos taurus), Ss (Su scrofa), Mm (Mus musculus).

Additional file 7 Percent frequency of repetitive elements flanking retroposed sequences and genes encoding glycolytic enzymes in the (A) human and (B) mouse genomes. Black bars represent the percent frequency of SINE, LINE and LTR elements flanking all intron-containing parent genes that encode glycolytic enzymes. Gray bars denote the percent frequency of SINE, LINE and LTR elements both upstream and downstream of retroposed sequences derived from these parent genes. White bars represent the genome average frequency of these elements, as determined by Waterston et al., 2002 [45].
Additional file $\mathbf{8}$ Retroposed and non-retroposed genes evolve at similar rates within each gene family. The figure shows the phylogenetic trees for five gene families (glyceraldehyde 3-phosphate dehydrogenase, pyruvate kinase, aldolase, phosphoglycerate mutase and hexokinase). h, denotes human genes; $m$, denote mouses genes. Black lines are used in branches for which we did not find evidence of retrotransposition. Red lines represent branches with evidence of retrotransposition. Numbers denote the average branch length since the primate/rodent split.

\section{Authors' contributions}

SAV developed and performed experiments, analyzed data, and drafted the manuscript. FPMV and DAO conceived of the study, participated in experimental design, and edited the manuscript. All authors read and approved the final manuscript.

\section{Acknowledgements}

Supported by the Eunice Kennedy Shriver NICHD/NIH through cooperative agreement U54 HD35041 as part of the Specialized Cooperative Centers Program in Reproduction and Infertility Research. We thank Tom Randall of the UNC-CH Center for Bioinformatics for technical assistance. We also thank Michael O'Rand and the Andrology Laboratory, Department of Obstetrics and Gynecology, University of North Carolina School of Medicine for the human sperm samples.

\section{Author Details}

'Department of Cell and Developmental Biology, University of North Carolina School of Medicine, Chapel Hill, NC 27599, USA, ${ }^{2}$ Department of Genetics, Carolina Center for Genome Sciences, University of North Carolina School of Medicine, Chapel Hill, NC 27599, USA, 3Laboratories for Reproductive Biology, Department of Pediatrics, University of North Carolina School of Medicine, Chapel Hill, NC 27599, USA and 4 Lineberger Comprehensive Cancer Center, University of North Carolina School of Medicine, Chapel Hill, NC 27599, USA

Received: 26 August 2009 Accepted: 6 May 2010

Published: 6 May 2010

\section{References}

1. Bunch DO, Welch JE, Magyar PL, Eddy EM, O'Brien DA: Glyceraldehyde 3phosphate dehydrogenase-S protein distribution during mouse spermatogenesis. Biol Reprod 1998, 58:834-841.

2. Welch JE, Schatte EC, O'Brien DA, Eddy EM: Expression of a glyceraldehyde 3-phosphate dehydrogenase gene specific to mouse spermatogenic cells. Biol Reprod 1992, 46:869-878.

3. Boer PH, Adra CN, Lau YF, McBurney MW: The testis-specific phosphoglycerate kinase gene $p g k-2$ is a recruited retroposon. Mol Cell Biol 1987, 7:3107-3112.

4. Vemuganti SA, Bell TA, Scarlett CO, Parker CE, de Villena FP, O'Brien DA: Three male germline-specific aldolase $A$ isozymes are generated by alternative splicing and retrotransposition. Dev Biol 2007, 309:18-31.

5. Mori C, Nakamura N, Welch JE, Gotoh H, Goulding EH, Fujioka M, Eddy EM: Mouse spermatogenic cell-specific type 1 hexokinase ( $m H k 1-s)$ transcripts are expressed by alternative splicing from the $m H k 1$ gene and the HK1-S protein is localized mainly in the sperm tail. Mol Reprod Dev 1998, 49:374-385.

6. Mori C, Welch JE, Fulcher KD, O'Brien DA, Eddy EM: Unique hexokinase messenger ribonucleic acids lacking the porin-binding domain are developmentally expressed in mouse spermatogenic cells. Biol Reprod 1993, 49:191-203.

7. Nakamura N, Shibata H, O'Brien DA, Mori C, Eddy EM: Spermatogenic cellspecific type 1 hexokinase is the predominant hexokinase in sperm. Mol Reprod Dev 2008, 75:632-640

8. Feiden S, Stypa H, Wolfrum U, Wegener G, Kamp G: A novel pyruvate kinase (PK-S) from boar spermatozoa is localized at the fibrous sheath and the acrosome. Reproduction 2007, 134:81-95.

9. Buehr M, McLaren A: An electrophoretically detectable modification of glucosephosphate isomerase in mouse spermatozoa. J Reprod Fertil 1981, 63:169-173.

10. Yakirevich $\mathrm{E}$, Naot $\mathrm{Y}$ : Cloning of a glucose phosphate isomerase/ neuroleukin-like sperm antigen involved in sperm agglutination. Biol Reprod 2000, 62:1016-1023. 
11. Russell DL, Kim KH: Expression of triosephosphate isomerase transcripts in rat testis: evidence for retinol regulation and a novel germ cell transcript. Biol Reprod 1996, 55:11-18.

12. Edwards $\mathrm{YH}$, Grootegoed JA: A sperm-specific enolase. J Reprod Fertil 1983, 68:305-310.

13. Gitlits VM, Toh BH, Loveland KL, Sentry JW: The glycolytic enzyme enolase is present in sperm tail and displays nucleotide-dependent association with microtubules. Eur J Cell Biol 2000, 79:104-111.

14. Force A, Viallard JL, Saez F, Grizard G, Boucher D: Electrophoretic characterization of the human sperm-specific enolase at different stages of maturation. J Androl 2004, 25:824-829.

15. Yamada S, Nakajima H, Kuehn MR: Novel testis- and embryo-specific isoforms of the phosphofructokinase- 1 muscle type gene. Biochem Biophys Res Commun 2004, 316:580-587.

16. Mukai C, Okuno M: Glycolysis plays a major role for adenosine triphosphate supplementation in mouse sperm flagellar movement. Biol Reprod 2004, 71:540-547.

17. Peterson RN, Freund M: Glycolysis by washed suspensions of human spermatozoa. Effect of substrate, substrate concentration, and changes in medium composition on the rate of glycolysis. Biol Reprod 1969, 1:238-246.

18. Williams AC, WC F: The role of glucose in supporting motility and capacitation in human spermatozoa. J Androl 2001, 22:680-695.

19. Danshina PV, Geyer CB, Dai Q, Goulding EH, Willis WD, Kitto GB, McCarrey $J R$, Eddy EM, O'Brien DA: Phosphoglycerate kinase 2 (PGK2) is essential for sperm function and male fertility in mice. Biol Reprod 2010, 82:136-145.

20. Miki K, Qu W, Goulding EH, Willis WD, Bunch DO, Strader LF, Perreault SD, Eddy EM, O'Brien DA: Glyceraldehyde 3-phosphate dehydrogenase-S, a sperm-specific glycolytic enzyme, is required for sperm motility and male fertility. Proc Natl Acad Sci USA 2004, 101:16501-16506.

21. Odet F, Duan C, Willis WD, Goulding EH, Kung A, Eddy EM, Goldberg E: Expression of the gene for mouse lactate dehydrogenase $C$ (Ldhc) is required for male fertility. Biol Reprod 2008, 79:26-34.

22. Curi SM, Ariagno JI, Chenlo PH, Mendeluk GR, Pugliese MN, Sardi Segovia LM, Repetto HE, Blanco AM: Asthenozoospermia: analysis of a large population. Arch Androl 2003, 49:343-349

23. Nielsen R, Bustamante C, Clark AG, Glanowski S, Sackton TB, Hubisz MJ, Fledel-Alon A, Tanenbaum DM, Civello D, White TJ, et al:: A scan for positively selected genes in the genomes of humans and chimpanzees. PLoS Biol 2005, 3:e170.

24. Swanson WJ, Vacquier VD: The rapid evolution of reproductive proteins. Nat Rev Genet 2002, 3:137-144.

25. Steinke $D$, Hoegg S, Brinkmann $H$, Meyer A: Three rounds (1R/2R/3R) of genome duplications and the evolution of the glycolytic pathway in vertebrates. BMC Biol 2006, 4:16.

26. McCarrey JR, Thomas K: Human testis-specific PGK gene lacks introns and possesses characteristics of a processed gene. Nature 1987 326:501-505

27. Welch JE, Brown PR, O'Brien DA, Eddy EM: Genomic organization of a mouse glyceraldehyde 3-phosphate dehydrogenase gene (Gapd-s) expressed in post-meiotic spermatogenic cells. Dev Genet 1995, 16:179-189.

28. Vandeberg JL, Cooper DW, Sharman GB, Poole WE: Somatic expression and autosomal inheritance of phosphoglycerate kinase B in kangaroos. Genetics 1980, 95:413-424

29. Piechaczyk M, Blanchard JM, Riaad-EI Sabouty S, Dani C, Marty L, Jeanteur P: Unusual abundance of vertebrate 3-phosphate dehydrogenase pseudogenes. Nature 1984, 312:469-471.

30. Tolan DR, Niclas J, Bruce BD, Lebo RV: Evolutionary implications of the human aldolase- $A,-B,-C$, and -pseudogene chromosome locations. Am J Hum Genet 1987, 41:907-924.

31. Vemuganti SA: Genomic and proteomic analysis of aldolase A retrogenes expressed during spermatogenesis. Journal of Andrology 2006, 27:39.

32. Cortinas MN, Lessa EP: Molecular evolution of aldolase A pseudogenes in mice: multiple origins, subsequent duplications, and heterogeneity of evolutionary rates. Mol Biol Evol 2001, 18:1643-1653.

33. Dupressoir A, Heidmann T: Germ line-specific expression of intracisternal A-particle retrotransposons in transgenic mice. Mol Cell Biol 1996, 16:4495-4503.
34. Branciforte D, Martin SL: Developmental and cell type specificity of LINE-1 expression in mouse testis: implications for transposition. Mol Cell Biol 1994, 14:2584-2592.

35. Ergun S, Buschmann C, Heukeshoven J, Dammann K, Schnieders F, Lauke H, Chalajour F, Kilic N, Stratling WH, Schumann GG: Cell type-specific expression of LINE-1 open reading frames 1 and 2 in fetal and adult human tissues. J Biol Chem 2004, 279:27753-27763.

36. Ostertag EM, DeBerardinis RJ, Goodier JL, Zhang Y, Yang N, Gerton GL, Kazazian HH Jr: A mouse model of human L1 retrotransposition. Nat Genet 2002, 32:655-660.

37. Babushok DV, Kazazian HH Jr: Progress in understanding the biology of the human mutagen LINE-1. Hum Mutat 2007, 28:527-539.

38. Vinckenbosch N, Dupanloup I, Kaessmann H: Evolutionary fate of retroposed gene copies in the human genome. Proc Natl Acad Sci USA 2006, 103:3220-3225.

39. Hendriksen PJ, Hoogerbrugge JW, Baarends WM, de Boer P, Vreeburg JT, Vos EA, Lende T van der, Grootegoed JA: Testis-specific expression of a functional retroposon encoding glucose-6-phosphate dehydrogenase in the mouse. Genomics 1997, 41:350-359.

40. Kleene KC, Mulligan E, Steiger D, Donohue K, Mastrangelo MA: The mouse gene encoding the testis-specific isoform of Poly $(A)$ binding protein (Pabp2) is an expressed retroposon: intimations that gene expression in spermatogenic cells facilitates the creation of new genes. $J \mathrm{Mo} / \mathrm{EvOl}$ 1998, 47:275-281.

41. Emerson JJ, Kaessmann H, Betran E, Long M: Extensive gene traffic on the mammalian X chromosome. Science 2004, 303:537-540.

42. Marques AC, Dupanloup I, Vinckenbosch N, Reymond A, Kaessmann H: Emergence of young human genes after a burst of retroposition in primates. PLOS Biol 2005, 3:e357.

43. Nishimune $\mathrm{Y}$, Tanaka $\mathrm{H}$ : Infertility caused by polymorphisms or mutations in spermatogenesis-specific genes. J Androl 2006, 27:326-334

44. Torgerson DG, Kulathinal RJ, Singh RS: Mammalian sperm proteins are rapidly evolving: evidence of positive selection in functionally diverse genes. Mol Biol Evol 2002, 19:1973-1980.

45. Waterston RH, Lindblad-Toh K, Birney E, Rogers J, Abril JF, Agarwal P, Agarwala R, Ainscough R, Alexandersson $M, A \cap P$, et al:: Initial sequencing and comparative analysis of the mouse genome. Nature 2002, 420:520-562.

46. Krisfalusi M, Miki K, Magyar PL, O'Brien DA: Multiple glycolytic enzymes are tightly bound to the fibrous sheath of mouse spermatozoa. Biol Reprod 2006, 75:270-278.

47. Vandeberg JL, Lee CY, Goldberg E: Immunohistochemical localization of phosphoglycerate kinase isozymes in mouse testes. J Exp Zool 1981, 217:435-441.

48. Couldrey C, Carlton MB, Ferrier J, Colledge WH, Evans MJ: Disruption of murine alpha-enolase by a retroviral gene trap results in early embryonic lethality. Dev Dyn 1998, 212:284-292.

49. Fundele $\mathrm{R}$, Winking $H$, IIImensee $K$, Jagerbauer EM: Developmental activation of phosphoglycerate mutase- 2 in the testis of the mouse. Dev Biol 1987, 124:562-566.

50. McCarrey JR, Berg WM, Paragioudakis SJ, Zhang PL, Dilworth DD, Arnold $\mathrm{BL}$, Rossi JJ: Differential transcription of pgk genes during spermatogenesis in the mouse. Dev Biol 1992, 154:160-168.

51. de Luis O, del Mazo J: Gene expression of mouse M1 and M2 pyruvate kinase isoenzymes correlates with differential poly[A] tract extension of their mRNAs during the development of spermatogenesis. Biochim Biophys Acta 1998, 1396:294-305.

52. Shima JE, McLean DJ, McCarrey JR, Griswold MD: The murine testicular transcriptome: characterizing gene expression in the testis during the progression of spermatogenesis. Biol Reprod 2004, 71:319-330.

53. Pavlicek A, Gentles AJ, Paces J, Paces V, Jurka J: Retroposition of processed pseudogenes: the impact of RNA stability and translational control. Trends Genet 2006, 22:69-73.

54. Goncalves I, Duret L, Mouchiroud D: Nature and structure of human genes that generate retropseudogenes. Genome Res 2000, 10:672-678.

55. Zhang Z, Harrison PM, Liu Y, Gerstein M: Millions of years of evolution preserved: a comprehensive catalog of the processed pseudogenes in the human genome. Genome Res 2003, 13:2541-2558.

56. Storey BT: Mammalian sperm metabolism: oxygen and sugar, friend and foe. Int J Dev Biol 2008, 52:427-437. 
57. Hubbard TJ, Aken BL, Beal K, Ballester B, Caccamo M, Chen Y, Clarke L, Coates G, Cunningham F, Cutts T, et al.: Ensembl 2007. Nucleic Acids Res 2007, 35:D610-617.

58. Thompson JD, Higgins DG, Gibson TJ: CLUSTAL W: improving the sensitivity of progressive multiple sequence alignment through sequence weighting, position-specific gap penalties and weight matrix choice. Nucleic Acids Res 1994, 22:4673-4680.

59. O'Brien DA: Isolation, separation, and short-term culture of spermatogenic cells. In Male Reproductive Toxicology Volume 3A. Edited by: Chapin RE, Heindel JJ. Academic Press, Inc; 1993:246-264. [Tyson CA Witschi H (Series Editor): Methods in Toxicology].

60. O'Brien DA, Gabel CA, Rockett DL, Eddy EM: Receptor-mediated endocytosis and differential synthesis of mannose 6-phosphate receptors in isolated spermatogenic and Sertoli cells. Endocrinology 1989, 125:2973-2984.

61. Steger K, Pauls K, Klonisch T, Franke FE, Bergmann M: Expression of protamine- 1 and -2 mRNA during human spermiogenesis. Mol Hum Reprod 2000, 6:219-225.

62. Giardine B, Riemer C, Hardison RC, Burhans R, Elnitski L, Shah P, Zhang Y, Blankenberg D, Albert I, Taylor J, et al.: Galaxy: a platform for interactive large-scale genome analysis. Genome Res 2005, 15:1451-1455.

63. Rice P, Longden I, Bleasby A: EMBOSS: the European Molecular Biology Open Software Suite. Trends Genet 2000, 16:276-277.

64. Smit A, Hubley R, Green P: RepeatMasker Open-3.0. 1996.

\section{doi: 10.1186/1471-2164-11-285}

Cite this article as: Vemuganti et al., Frequent and recent retrotransposition of orthologous genes plays a role in the evolution of sperm glycolytic enzymes BMC Genomics 2010, 11:285

Submit your next manuscript to BioMed Central and take full advantage of:

- Convenient online submission

- Thorough peer review

- No space constraints or color figure charges

- Immediate publication on acceptance

- Inclusion in PubMed, CAS, Scopus and Google Scholar

- Research which is freely available for redistribution

Submit your manuscript at www.biomedcentral.com/submit 